\title{
Fair Play - ethische Grundlagen und pädagogische Potenziale
}

Marcel Reinold, Justus Kalthoff

\section{Abstract}

Fair Play stellt einen zentralen Wert des Olympismus, der Olympischen Erziehung und des Sports insgesamt dar. Bei einem sportlichen Wettbewerb werden ganz bestimmte Fähigkeiten verglichen und dies ist nur dann möglich, wenn alle Wettbewerbsteilnehmer"innen die gleichen Herausforderungen zu bewältigen und die gleichen Chancen auf den Sieg haben. Fair Play definiert als Chancengleichheit kann daher als die Conditio sine qua non für sportliche Leistungsvergleiche angesehen werden.

Im Hinblick auf Fair-Play-Erziehung sind die Olympischen Spiele und der Sport insgesamt ein Feld mit außergewöhnlichem Potential. Durch seine agonale Grundstruktur und seinem Spiel- bzw. Sonderweltcharakter kann er als ein moralisches Labor begriffen werden, in dem sich - verhältnismäßig konsequenzlos - experimentieren lässt. Indem moralische Konfliktlagen vom Lehrpersonal situativ aufgegriffen bzw. pädagogisch-didaktisch inszeniert werden, entstehen Lerngelegenheiten, in denen (un-)moralisches Handeln hautnah erprobt, erfahren und reflektiert werden kann.

\section{Einleitung: Olympismus, Olympische Erziehung und Fair Play}

Der Olympismus stellt ein Ensemble unterschiedlicher Werte und Ziele dar. Aufbauend auf den Schriften von Pierre de Coubertin, dem Initiator der Olympischen Spiele der Neuzeit, arbeitet der Sportphilosoph Hans Lenk (1972) insgesamt elf Olympische Werte und Ziele heraus: Erstens ließen sich die Spiele als eine kultisch-religiöse Feier charakterisieren, die Coubertin als „religio athletae“ bezeichnete. Zweitens sollten die Spiele keine rein körperliche Angelegenheit darstellen, sondern künstlerisch und geistig mitgestaltet werden. Drittens solle der Olympische Sport von der Idee der Elite und Chancengleichheit geprägt sein. Viertens gehe es dabei einerseits um Höchstleistung und Wettkampf. Andererseits solle der Wettkampf, fünftens, vom Geist des Fair Play bzw. - wie Coubertin 
sich ausdrückte - von "Ritterlichkeit“ durchdrungen sein. Sechstens fänden die Spiele in einem vierjährigen Rhythmus unter der Idee des „Burgfriedens“ statt. Siebtens handele es sich um internationale Spiele mit dem Ziel der Völkerverständigung. Achtens sollten alle Sportarten zusammen eine Gemeinschaft bilden. Neuntens würde dem Amateurgedanken ein zentraler Stellenwert beigemessen. Zehntens solle die Olympische Bewegung unabhängig sein. Und elftens diene die Antike als Vorbild des modernen Olympischen Sports.

Die Olympische Idee war somit von vornherein auch eine Erziehungsidee. Der Sportpädagoge Ommo Grupe (2013, S. 13 ff.) unterscheidet dabei fünf Grundprinzipien der Olympischen Erziehung. Erstens das Prinzip der Leib-Seele-Einheit bzw. der ganzheitlichen Erziehung des Menschen; zweitens das Ziel der Selbstgestaltung bzw. Selbstvollendung; drittens das Ideal des Amateurismus; viertens die Bindung des Sports an ethische Regeln und Grundsätze, wobei vor allem an Fair Play zu denken ist; und fünftens die Friedensidee des Olympischen Sports. Der Olympiahistoriker Norbert Müller (1998, S.393) hat diesen Grundprinzipien mit der „Förderung emanzipatorischer Entwicklungen im und durch Sport“ ein sechstes Prinzip hinzugefügt.

In diesem Beitrag soll nicht dieses gesamte Ensemble an Werten, Zielen und Grundprinzipien des Olympismus bzw. der Olympischen Erziehung diskutiert werden. Vielmehr liegt der Fokus auf Fair Play. Dafür sprechen in erster Linie drei Gründe: Erstens haben einige der genannten Werte, Ziele und Grundprinzipien im Laufe der Zeit an Relevanz verloren oder wurden sogar gänzlich aufgegeben. So wurde etwa im Zuge der überwältigenden Prozesse der Professionalisierung und Kommerzialisierung des Sports ab den 1960er Jahren der Amateurismus zunehmend aufgeweicht und schließlich mit der Liberalisierung der Zulassungsregel auf dem Olympischen Kongress 1981 in Baden-Baden weitgehend abgeschafft (Llewellyn \& Gleaves, 2016, S. 142 ff.). Hinzu kommt, zweitens, dass manche von Coubertins Zentralbegriffen wie Ritterlichkeit oder religio athletae als moralische Werte antiquiert erscheinen. Drittens sind bestimmte Werte für den Olympischen Sport zentraler als andere. So räumt Lenk (1972, S. 277 f.) dem Konkurrenz-, Wettkampf- und Leistungsprinzip sowie der Idee der Chancengleichheit und des Fair Play eine konstitutive Funktion ein, während etwa dem vierjährigen Rhythmus der Spiele eher eine regulative Funktion zukomme, weil dieser durchaus auch anders sein könne, ohne dass sich der Olympische Sport im Kern verän- 
dere. ${ }^{1}$ Die Bedeutung von Fair Play zeigt sich auch darin, dass es als eines von fünf Olympischen Erziehungsthemen im Olympic Values Education Programme des IOC (2017, S. 31) berücksichtigt wird. Heutzutage wird Fair Play sogar von vielen Expert*innen als der zentrale Wert des Sports angesehen (Lenk, 2004, S. 119). Nicht zuletzt sind seine Ursprünge genuin im Sport zu verorten (Guttmann, 1987). In modernen Leistungsgesellschaften wird Fair Play jedoch weit über den Sport hinaus überall dort in Anspruch genommen, wo Menschen zueinander in Konkurrenz stehen und Agonalität geregelt werden muss (Sandel, 2020, S. 53 ff.). Aus dem Kanon der Olympischen Grundprinzipien, Ziele und Werte rückt dieser Beitrag daher Fair Play in den Mittelpunkt.

Zuerst erfolgt dabei die Klärung grundlegender, theoretisch-methodischer Fragen zur Sportethik als derjenigen sportwissenschaftlichen Teildisziplin, in deren Kompetenzbereich die Hinterfragung und Begründung moralischer Werte im Sport fällt. Unter Rekurs auf aktuelle Theoriedebatten in der internationalen Sportethik schlagen wir anschließend eine internalistische Konzeptionalisierung des Fair-Play-Begriffs vor. Abschließend wird der pädagogische Anspruch des Olympismus aufgegriffen und das grundsätzliche Potenzial des Sports für die Fair-Play-Erziehung ausgelotet.

\section{Gegenstand und Methoden der Sportethik}

Zunächst geht es um die Klärung der Fragen, mit was sich die Sportethik beschäftigt (Gegenstand) und wie sie zu ihren Erkenntnissen kommt (Methode).

Bei vielen zentralen Fragen im Sport geht es weniger um Ist-Zustände als vielmehr um Soll-Zustände. Letztere bezeichnet man als Normen. Zu Fragen wäre etwa: Wie soll gehandelt werden, wenn ein Regelverstoß vorliegt? Die systematische Klärung und Begründung dieser normativen (Wert-)Fragen fällt in den Bereich der Sportethik als Teildisziplin der Sportphilosophie. Inhaltlich betreffen diese Fragen zwar den Sport, die grundlegenden Theorien und Methoden kommen jedoch meist aus der Mutterwissenschaft - der allgemeinen Ethik, die wiederum eine Teildiszi-

1 Die Unterscheidung zwischen regulativ und konstitutiv geht auf den Sprachphilosophen John Searle zurück und wurde in der Sportphilosophie zur Differenzierung von Regeln verschiedentlich aufgegriffen (Gleaves, 2014). 
plin der Philosophie darstellt (Meinberg, 1998, S. 499). Insofern macht es Sinn, zunächst einen Blick auf die Mutterwissenschaft zu werfen.

Die Philosophin Annemarie Pieper (2017, S. 10) definiert den Gegenstandsbereich der Ethik folgendermaßen:

„Die Ethik hat es mit menschlichen Handlungen zu tun. [...] Sie fragt nach diesem qualitativen Moment, das eine Handlung zu einer moralisch guten Handlung macht [...]. Die Ethik beschäftigt sich auf methodische Weise mit ihrem Gegenstand - mit moralischen Handlungen -, da sie zu argumentativ begründeten Ergebnissen gelangen will [...]. [Der Ethik geht es demnach um Aussagen], die nicht bloß subjektiv gültig, sondern als intersubjektiv verbindlich ausweisbar sind.“

In der Ethik geht es also um menschliche Handlungen. Das finale Erkenntnisinteresse liegt dabei aber - anders als beispielsweise in der Psychologie - weder in der Beschreibung noch in der Erklärung. Vielmehr geht es der Ethik um die moralische Qualität von Handlungen.

\section{Ethik}

Im Unterschied zu einer empirischen Wissenschaft wie der Psychologie fragt die Ethik letztlich nicht nach Ist-Zuständen und deren Erklärung, d.h. nicht danach, wie Menschen tatsächlich handeln und warum sie so handeln wie sie handeln, sondern nach Soll-Zuständen, d.h. da-nach, wie Menschen handeln sollen.

Zu ihren Ergebnissen gelangt die Ethik dabei auf methodische Weise: Es handelt sich nicht um subjektive Meinungen, die sozusagen ,je nach Interessenlage und Gusto' auch ganz anders hätten ausfallen können, sondern vielmehr um argumentativ begründete Aussagen, die intersubjektiv nachvollziehbar sind. Definiert man nun den Gegenstand der Sportethik in Anlehnung an die Definition der allgemeinen Ethik von Pieper, so geht es hierbei um die argumentativ begründete und intersubjektiv nachvollziehbare Bewertung der moralischen Qualität von Handlungen im Feld des Sports. Ganz ähnlich definieren auch die Philosophen Jürgen Court und Volker Gerhardt (1992, S. 428) die Sportethik als die „Analyse und Bewertung moralischer Einstellungen und Vollzüge im Feld des Sports“.

Um die Nachvollziehbarkeit einzelner Denkschritte zu erhöhen, können komplexe ethische Argumentationen in sogenannte Syllogismen umformuliert werden. Ein Syllogismus ist ein seit Aristoteles (384-322 v. Chr.) bekannter, logischer Schluss, der aus mehreren Prämissen (Voraus- 
setzungen) und einer Konklusion (Schlussfolgerung) besteht (Tetens, 2006, S. 22 ff.). Im Folgenden ein einfaches Beispiel. Nehmen wir an, eine Sportpolitikerin ${ }^{2}$ macht sich für den Anti-Doping-Kampf stark und argumentiert in einer Rede folgendermaßen:

Meine Damen und Herren, ich bin gegen Doping, weil Doping im Sport unfair ist. Des Weiteren bin ich der Meinung, dass wir alles verbieten sollen, was unfair ist. Das ist der Grund, warum es ein Dopingverbot gibt und dies auch so bleiben soll.

Folgende sind die tragenden Prämissen der Argumentation der Sportpolitikerin: Erstens wird festgestellt, dass Doping im Sport unfair sei, und zweitens, dass alles, was unfair sei, verboten sein solle. Daraus wird geschlossen, dass auch Doping verboten sein solle. In Form eines Syllogismus dargestellt ergäbe sich folgende Argumentation:

\begin{tabular}{|l|l|}
\hline Erste Prämisse: & Doping im Sport ist unfair. \\
\hline Zweite Prämisse: & Alles, was unfair ist, soll verboten sein. \\
\hline Konklusion: & Doping soll verboten sein.
\end{tabular}

Logisch betrachtet handelt sich um einen gültigen Schluss, der notwendigerweise wahr ist, wenn die beiden Prämissen auch wahr sind. Dabei handelt es sich bei der ersten Prämisse um eine deskriptive und bei der zweiten um eine präskriptive Prämisse. Eine deskriptive Prämisse ist eine Ist-Aussage („ist unfair“), die etwas beschreibt (i.e. „deskriptiv“), eine präskriptive Prämisse hingegen eine Soll-Aussage („soll verboten werden“), die etwas vorschreibt (i.e. "präskriptiv“). Die Konklusion wiederum ist präskriptiv („soll verboten sein“). Argumentationstheoretisch gesehen wird bei einer ethischen Argumentation stets eine deskriptive Prämisse mit

2 Über die Verwendung des Gendersternchens hinaus wird in beispielhaften Ausführungen bei Personenbezeichnungen aus Gründen der Lesbarkeit an manchen Stellen die weibliche, an anderen Stellen die männliche Form verwendet. Wenn daher beispielsweise von der "Sportpolitikerin“ oder der "Athletin“ etc. gesprochen wird, ist somit auch stets der "Sportpolitiker" oder der "Athlet" etc. mitgemeint. Die gewählte Geschlechtsbezeichnung ist also zufällig bzw. stellvertretend und nicht explizit bzw. inhaltlich begründet. Zwar machen empirische Untersuchungen deutlich, dass der Faktor Geschlecht beim subjektiven Verständnis von Fair Play durchaus eine Rolle spielt (Gaum, 2017, S. 352 \& 356; Pilz, 1995, S. 177, 195; Kähler, 1985, S. 145). Im Rahmen dieses Überblicksartikels können solche geschlechtsspezifischen Unterschiede jedoch nicht weiter behandelt werden. 
einer präskriptiven verbunden und so ein ethisches Urteil gefällt (die präskriptive Konklusion) (Dietrich, 2009, S. 214f.): Weil Doping im Sport unfair ist (deskriptive Prämisse) und weil alles, was unfair ist, verboten sein soll (präskriptive Prämisse), soll Doping verboten sein (präskriptive Konklusion).

Auf die Unterscheidung von deskriptiv und präskriptiv wird im Abschnitt „Fair Play aus sportethischer Perspektive“ zurückzukommen sein. Zunächst soll aber die Frage geklärt werden, wie ethische Argumentationen geprüft und potenziell entkräftet werden können. Dazu muss erstens die Gültigkeit des logischen Schlusses überprüft werden. Logisch ungültig wäre die Argumentation beispielsweise dann, wenn die zweite Prämisse lauten würde: „Vieles, was unfair ist im Sport, soll verboten werden." Die Relativierung „vieles" bedeutet eben, dass nicht alles, was unfair ist, verboten werden soll. $\mathrm{Zu}$ den nicht zu verbietenden unfairen Handlungen könnte dann potenziell auch Doping gehören, weshalb das pauschale Urteil, dass Doping verboten werden soll, nicht mehr logisch aus dieser veränderten Prämissenlage folgt. In der ursprünglich skizzierten Form (,alles, was unfair ist im Sport, soll verboten sein“) ist die Argumentation jedoch logisch gültig. Eine zweite Möglichkeit, die Argumentation zu entkräften, besteht darin, die Prämissen anzugreifen. Bezüglich der ersten Prämisse ist dann etwa zu hinterfragen, ob Doping im Sport denn wirklich (immer) unfair ist. Ist Doping beispielsweise auch dann unfair, wenn sich alle Sportler innerhalb eines Wettbewerbs dopen? Von einseitiger Vorteilsnahme kann dann jedenfalls nicht mehr die Rede sein.

\section{Fair Play aus sportethischer Perspektive: Definitionen}

In seiner Monographie zum Thema Fairness trägt der US-amerikanische Politikwissenschaftler und Philosoph Craig Carr (2000, S.2) sechs verschiedene Fairnessdefinitionen aus der Literatur zusammen. Nach dem Verständnis von manchen Autor*innen zeichne sich faires Handeln erstens dadurch aus, dass andere nicht benachteiligt würden. Zweitens werde Fairness teilweise als Unparteilichkeit, Objektivität und Neutralität definiert. Drittens verwendeten manche den Fairnessbegriff gleichbedeutend mit dem Begriff der Chancengleichheit. Eine vierte Definition besage, dass Fairness in der Gleichbehandlung von Gleichem bestehe. Fünftens werde Fairness teilweise in der Befolgung der formal festgehaltenen Regeln gesehen. Und sechstens sei Fairness dann gegeben, wenn andere mit Achtung und Respekt behandelt würden. Carrs Differenzierungen sind nicht erschöpfend. Hinzuzufügen sind zumindest die Defini- 
tion von Fairness als die Einhaltung eines (explizit oder implizit geschlossenen) Vertrags (Butcher \& Schneider, 2001, S. 22; Reinold \& Kalthoff, 2019, S. $11 \mathrm{ff}$.) und die Definition von Fairness als Fairness gegenüber sich selbst. Fairness verstanden als Fairness gegenüber sich selbst zielt wesentlich auf einen fairen Umgang mit dem eigenen Körper, der darauf angelegt ist, dass Athlet"innen nicht nur zum gegenwärtigen Zeitpunkt, sondern auch zukünftig einen gesunden Körper haben werden (Siep, 1995, S. 98 ff.; Caysa, 2004, S. 149 ff.).

\section{Fairness und Fair Play}

Die Begriffe „Fairness“ und „Fair Play“ werden in der Literatur oftmals synonym verwendet. Der Unterschied besteht in erster Linie darin, dass der Begriff des „Fair Play“ den Spielbegriff beinhaltet und damit implizit auf Fairness in spielerischen und sportlichen Zusammenhängen verweist, während der Begriff der „Fairness“ auch stärker in Zusammenhängen außerhalb von Spiel und Sport gebraucht wird.

Im Rahmen dieses Artikels kann nicht mit allen genannten Definitionen weitergearbeitet werden. Im Folgenden wird daher zunächst ein bestimmter Fairnessbegriff, nämlich Fairness definiert als die Befolgung der formal festgehaltenen Regeln, herausgegriffen und auf eine spezifische Thematik, die Dopingthematik, angewandt. Faires Handeln im Sport sei also definiert als Handeln in Übereinstimmung mit den formal festgehaltenen Regeln. Im Umkehrschluss sind alle Handlungen genau dann als unfair zu beurteilen, wenn sie formal verboten sind. Daraus lässt sich als erste (deskriptive) Prämisse folgende Definition formulieren: Unfair sind Handlungen genau dann, wenn sie verboten sind. Prüft man nun auf der Grundlage dieser Definition, ob Doping unfair ist, so muss man auf der Ebene der Fakten prüfen, ob Doping formal verboten ist. Ein Blick in die Regelwerke zeigt, dass ein Dopingverbot besteht. Daher lässt sich als zweite (deskriptive) Prämisse feststellen, dass Doping unfair ist. Man sieht an diesem Beispiel gut, welch wichtige Rolle Fakten beim ethischen Argumentieren spielen: In diesem Fall wird durch einen Blick in die Regelwerke auf rein deskriptiver Ebene etwas festgestellt und so eine deskriptive Prämisse formuliert. Des Weiteren lässt sich im Anschluss an die Überlegungen des Sportphilosophen Bernard Suits (2004) und des Linguisten Hans Jürgen Heringer (1995, S. 58 ff.) argumentieren, dass ein Spiel nicht richtig gespielt werden kann, wenn sich die Teilnehmer nicht an die formalen Regeln halten. Ein Fußballspiel etwa konstituiert sich dadurch, dass der Ball (mit Ausnahme des Torwarts) nicht mit der Hand 
gespielt werden darf. Bei Nichteinhaltung dieser Regel spielen die Spieler*innen schlicht nicht (mehr) Fußball. Wiederum lässt sich eine (diesmal präskriptive) Prämisse formulieren, die besagt, dass Sportler*innen regelwidrige und damit gleichzeitig unfaire Handlungen unterlassen sollen, wenn sie ein bestimmtes Spiel wirklich spielen wollen. Aus diesen drei Prämissen lässt sich - logisch gültig - die (präskriptive) Schlussfolgerung ziehen, dass Sportler*innen nicht dopen sollen. In Form eines Syllogismus dargestellt ergibt sich also folgende Argumentation:

\begin{tabular}{|c|c|}
\hline Erste Prämisse: & $\begin{array}{l}\text { Unfair sind Handlungen genau dann, wenn } \\
\text { sie formal verboten sind. }\end{array}$ \\
\hline Zweite Prämisse: & Doping ist formal verboten. \\
\hline $\begin{array}{l}\text { Zwischenkonklusion } \\
\text { und dritte Prämisse: }\end{array}$ & Doping ist unfair. \\
\hline Vierte Prämisse: & $\begin{array}{l}\text { Sportler*innen sollen unfaire Handlungen } \\
\text { unterlassen. }\end{array}$ \\
\hline Konklusion: & Sportler*innen sollen nicht dopen. \\
\hline
\end{tabular}

Da der Schluss logisch gültig ist, kann die Argumentation nur durch die Infragestellung der Prämissen angegriffen werden. Wenden wir uns diesbezüglich zunächst der zweiten Prämisse zu: Doping ist deswegen unfair, weil es durch Regeln verboten ist. Wenn Doping hingegen nicht verboten wäre, so wäre es auch nicht unfair. Dies ist eine Folgerung aus der ersten Prämisse, die Handlungen genau dann - und das bedeutet nur dann - als unfair klassifiziert, wenn sie formal verboten sind. Eine Legalisierung hätte also unmittelbare Auswirkungen auf die zweite Prämisse und damit auf die gesamte Argumentation. Die dritte Prämisse besagt nämlich, dass Sportler*innen lediglich unfaire Handlungen unterlassen sollen. Mit seiner Legalisierung fiele Doping jedoch nicht mehr in die Kategorie der unfairen Handlungen. Insofern könnte nicht mehr geschlossen werden, dass Sportler*innen nicht dopen sollen. Allerdings könnte auch nicht geschlossen werden, dass Sportler*innen dopen sollen, denn darüber wie Handlungen moralisch zu bewerten sind, die nicht in die Kategorie verboten bzw. unfair fallen, sagt die Argumentation nichts.

Man sieht an diesem Beispiel, dass die Definition von „unfair“, wie sie in der ersten Prämisse vorgenommen wird, möglicherweise in zweierlei Hinsicht unzureichend ist: Es könnte nämlich, erstens, auch Handlungen geben, die zwar nicht explizit verboten, aber dennoch als unfair zu 
beurteilen sind. Handelt beispielsweise eine Rennradfahrerin bei Olympischen Spielen nicht auch unfair, wenn sie das Pech einer unglücklich gestürzten Konkurrentin ausnutzt, um das Tempo zu forcieren, obwohl formal keine Regel existiert, die dies verbietet? Tatsächlich sieht man bei Radrennen oft, wie das Fahrerinnenfeld bei einem Sturz das Tempo drosselt, um der gestürzten Konkurrentin den Anschluss zu ermöglichen. Es handelt sich um eine informelle Konvention (eine stillschweigende Übereinkunft, ein , ungeschriebenes Gesetz ${ }^{6}$ ) innerhalb des Fahrerinnenfeldes, die besagt, dass bestimmte Situationen wie Stürze oder auch technische Defekte nicht zum eigenen Vorteil ausgenutzt werden sollen, und an die sich die Fahrerinnen normalerweise halten. Sollte man solche Handlungen nicht ebenfalls als fair beurteilen, obwohl hierbei gar keine formal existierende Regel eingehalten wird? Der Sportphilosoph Hans Lenk (2004, S. 120 ff.) verwendet für diese Art von Fairness den Begriff der „informellen Fairness" - in Abgrenzung zur „formellen Fairness“, die als die Einhaltung der formalen Regeln definiert wird und dem oben dargestellten Syllogismus zugrunde liegt. Zweitens stellt sich die Frage, ob es nicht auch unfaire Regeln geben kann, deren Einhaltung dann wiederum unfaire Handlungen nach sich ziehen würden. Man denke hierbei etwa an die Amateurregeln früherer Zeiten, die zum Beispiel Sportlern aus der Arbeiterklasse die Teilnahme an bestimmten Wettbewerben verweigerten (Llewellyn \& Gleaves, 2016), oder auch geschlechterdiskriminierende Regeln, die Frauen von bestimmten Sportarten ausschlossen (Günter, 2005). Hier stellt sich das grundsätzliche Problem, dass Regeln nicht mehr als fair oder unfair hinterfragt werden können, wenn Fairness a priori über Regelgebundenheit definiert wird.

Definiert man Fairness nun stattdessen über den Begriff der Chancengleichheit, so lassen sich die genannten Probleme vermeiden. Erstens können dann auch Regeln als fair oder unfair hinterfragt werden. Und zweitens können Handlungen, die zwar formell keinen Regelverstoß darstellen, aber einem informellen Fairnessverständnis widersprechen, als unfair beurteilt werden. Jedoch weist auch diese Definition Schwierigkeiten auf: So stellt sich die Frage, ob Doping auch dann noch als unfair verurteilt werden kann, wenn sich alle Sportler*innen innerhalb eines Wettbewerbs dopen würden. Von einer einseitigen (und damit die Chancengleichheit beeinträchtigenden) Vorteilsnahme könnte dann jedenfalls nicht mehr die Rede sein (Reinold, 2016, S. 159 ff.). Dennoch wird sich im folgenden Abschnitt zeigen, dass die Definition von Fairness als Chancengleichheit Sinn macht, und zwar vor allem dann, wenn man 
den Fairnessbegriff internalistisch, d.h. aus einer bestimmten normativen Theorie von Sport heraus entwickelt. ${ }^{3}$

\section{Warum fair sein im Sport? Internalistische Theorieansätze und moralische Konsequenzen}

Leistungsvergleiche werden erst dann möglich, wenn alle Wettkampfteilnehmer"innen dieselbe Distanz zurücklegen und dafür nur dasselbe (oder zumindest sehr ähnliches) Material benutzen dürfen (zum Beispiel Skier beim Skifahren, Fahrräder bei Radrennen etc.). Dafür gibt es Regeln, die gleiche Ausgangsbedingungen herstellen (Loland, 2010, S. 119). Gleichzeitig konstituieren diese Regeln die jeweilige Sportart bzw. Disziplin: Ein 400-Meter-Lauf wird zum 400-Meter-Lauf dadurch, dass alle Athlet*innen 400 Meter laufen. Der „Witz des Spiels“ (Heringer, 1995, S. 58) wäre zerstört, wenn beispielsweise einer der Konkurrenten die Strecke abkürzen oder mit dem Fahrrad fahren würde. Solche Regelbrüche machen das ursprüngliche Ziel, 400 Meter zu laufen, schlicht unerreichbar (Suits, 2004, S. 32). Abstrakt gesehen schaffen Regeln die Herausforderungen, für deren Bewältigung absichtlich nur bestimmte und oftmals vergleichsweise ineffiziente Mittel zugelassen sind (Gleaves, 2014, S.368): Man läuft möglichst schnell, um die ganze Bahn anstatt effizienter Weise den Weg quer über das Innenfeld einzuschlagen oder Fahrrad zu fahren (Suits, 2004, S.30). Dass dies (normalerweise) nicht geschieht, liegt an den Regeln, die für den 400-Meter-Lauf formuliert wurden. Die konstitutive Funktion von Regeln hat manche Sportphilosoph*innen dazu veranlasst, Regeln als den Kern dessen zu begreifen, was Sport grundsätzlich ausmacht. Solche Positionen werden unter dem Begriff ,Formalismus zusammengefasst. Dieser Begriff verweist darauf, dass Sport sich über seine formale Struktur, d.h. die formalisiert festgehaltenen Regeln, definiert (Triviño, 2014, S. 358).

Entwickelt man den Fairnessbegriff aus dieser streng formalistischen Position heraus, so kommt man zum Begriff der ,formellen Fairness

3 Sportethische Positionen, welche davon ausgehen, dass Werte und Normen aus dem Sport selbst heraus entwickelt werden können, lassen sich unter dem Begriff des „Internalismus“ zusammenfassen. Innerhalb des Internalismus wiederum lassen sich Formalismus, Konventionalismus und breiter Internalismus differenzieren. Der Externalismus hingegen bezeichnet Positionen, bei denen Werte und Normen von außen an den Sport herangetragen werden. Siehe zu dieser Differenzierung ausführlich Simon (2015, S. 22 ff.) sowie López Frías (2017, S. 22 f.). 
(Lenk, 2004, S. 120 ff.). Die Probleme eines solchen Fairnessbegriffs wurden bereits erörtert: Erstens kann es durchaus auch unfaire Regeln geben. Regeln sind also nicht schon deswegen fair, weil sie existieren, sondern existierende Regeln können auch moralisch falsch sein. Zweitens weisen Positionen, die in der Sportethik unter dem Begriff des Konventionalismus zusammengefasst werden, darauf hin, dass sich Sport nicht nur über die formalen Regeln definiert, sondern auch wesentlich über informelle Konventionen, d.h. die ungeschriebenen Gepflogenheiten, Werte und Normen, ohne die Handlungen im Sport nicht zu verstehen sind (D’Agostino, 1981, S. 14 ff.; Morgan, 2015).

Hingegen behauptet ein breiter angelegter Internalismus - in der einschlägigen englischsprachigen Literatur ist von „broad internalism“ die $R^{2} e^{4}$-, dass weder Regeln noch Konventionen den Kern von Sport ausmachen. Vielmehr wird der Fokus auf die Interpretation von Sport bzw. bestimmten Sportarten gelenkt. Was macht eine bestimmte Sportart im Kern aus, was ist das Ziel des Spiels und welche Regeln und Konventionen ergeben diesbezüglich am meisten Sinn (Simon, 2010, S. 52; Russel, 1999, S. 35)? Konkret gefragt: Warum müssen die 400 Meter notwendigerweise gelaufen werden, obwohl es schnellere Mittel und Wege gibt, im Ziel anzukommen, und warum warten Radfahrer*innen bei technischen Defekten ihrer Konkurrent"innen aufeinander, obwohl sie dies eigentlich nicht müssten? Anhänger*innen des breiten Internalismus antworten darauf, dass dadurch die für den jeweiligen Sport relevanten Herausforderungen überhaupt erst entstehen und damit sportliche Leistung überhaupt erst zum Vorschein gebracht wird. Wer Läufern hingegen erlaubt, das Auto zu nehmen, misst nicht läuferische Leistung, sondern Motorleistung und vielleicht auch fahrerisches Können. Ähnliches lässt sich bei der Missachtung bestimmter ungeschriebener Konventionen beobachten: Wenn Radfahrer*innen die technischen Defekte ihrer Konkurrent*innen während eines Wettkampfes ausnützen, dann entscheiden weniger sportliche Fähigkeiten, sondern vielmehr technisches Equipment und Zufälle die Radrennen. Der Sinn des Lauf- bzw. Radsports liegt aber darin, ein Ziel auf ganz bestimmte Weise zu erreichen und zwar so, dass die spezifischen

4 Von einem „breiten Internalismus“ wird deswegen gesprochen, weil diese Position über den engen Fokus des Formalismus auf Regeln bzw. des Konventionalismus auf Konventionen hinausgeht. Zum breiten Internalismus siehe in erster Linie die Arbeiten von Simon (2000, 2015), Torres (2000), Russell (1999) und Morgan (2012). Zur Abgrenzung von Formalismus, Konventionalismus und breitem Internalismus sowie für weitere Differenzierungen innerhalb des breiten Internalismus siehe Simon $(2000,2014)$ sowie López Frías (2017). 
sportlichen Fähigkeiten, d.h. Laufen bzw. Radfahren, getestet werden. Das ist das, worum es im Lauf- bzw. Radsport im Kern geht, und das durch die Einhaltung bestimmter geschriebener Regeln und ungeschriebener Konventionen aufrechterhalten werden soll.

Auf grundlegender Ebene interpretieren Anhänger*innen eines breiten Internalismus Leistungs- und Wettkampfsport als ein Streben nach Leistung durch spezifische Herausforderungen (Boxill, 2003, S. 1 f.; Russel, 2014, S. 228). Das Austesten körperlicher Leistungsfähigkeit wird durch die Anerkennung künstlicher Beschränkungen, die die effektivsten Wege zur Zielerreichung ausschließen, allererst möglich. Egal, ob es sich dabei um formalisierte Regeln oder informelle Konventionen handelt: In einem Sport, in dem ganz bestimmte Fähigkeiten getestet werden sollen, müssen die Regeln und Konventionen so beschaffen sein, dass das getestet wird, was getestet werden soll (Campos, 2014, S. 370; Fraleigh, 2014, S. 342). Das ist von Sportart zu Sportart unterschiedlich: Beim Laufsport sind es die läuferischen Fähigkeiten, beim Basketball Fähigkeiten wie Dribbling, Passspiel und Wurf. Würde man beim Basketball beispielsweise eine Regel einführen, die das Dribbling verbietet, so würde man das Spiel in der Weise verändern, dass Raumgewinne nur noch über Passspiel (und eben nicht mehr über Dribbling) möglich werden. Da Dribblings somit nicht mehr Teil des Basketballspiels wären, würden sie als eine spezifische Fähigkeit von Basketballspieler*innen auch keine Rolle mehr spielen. Ob eine solche Regeländerung Sinn macht, hängt von unserer Idee von Basketball ab.

Die Frage, was die Grundidee des jeweiligen sportlichen Wettkampfes ist und welche Fähigkeiten dabei getestet werden sollen, stellen also die zentralen Ausgangsfragen für die Bewertung von Regeln, Konventionen und Handlungen dar. Hinzu kommt jedoch noch ein weiterer Aspekt, bei dem Fairness eine wesentliche Rolle spielt: Wettkampfsport ist ein auf Leistungsvergleich angelegtes Unternehmen, bei dem die unterschiedlichen sportlichen Fähigkeiten der Wettbewerbsteilnehmer*innen zutage treten und sich in der Platzierung widerspiegeln sollen (Ott, 2004, S. 138). Dies hat zwei moralische Implikationen: Erstens müssen Sportler*innen ihren Konkurrent*innen mit Anerkennung, Achtung und Respekt begegnen. Trotz der notwendigen Rivalität braucht nämlich jede*r Sportler*in notwendigerweise wettbewerbsfähige Konkurrent*innen, um im sportlichen Vergleich wirklich siegen zu können (Gerhardt, 1997, S. 198). Diese Einsicht schließt nicht nur aus, dass Konkurrent“innen absichtlich verletzt werden, um zu gewinnen (Simon, 2000, S. 10). Das Beispiel der wartenden Radfahrerinnen zeigt außerdem, dass Sportlerinnen bisweilen sogar so weit gehen und unglücklich gestürzte Konkur- 
rentinnen aktiv in eine wettbewerbsfähige Lage zurückversetzen, damit wieder ein herausfordernder Wettkampf möglich wird (Butcher \& Schneider, 2001, S. 41). Ein gelingender sportlicher Wettbewerb ist daher nicht nur eine kompetitive, sondern wesentlich auch eine kooperative Angelegenheit (Gaffrey, 2015, S. 290; Simon, 2014, S. 92). Ein Fairnessbegriff, der auf Anerkennung, Achtung und Respekt vor dem Gegner zielt, wurzelt in dieser Einsicht (Heringer, 1995, S. 58). Gleichzeitig lassen sich diese Werte nicht nur aus dem Sport ableiten. Es handelt sich vielmehr um allgemein anerkannte gesellschaftliche Werte, die sich letztlich mit der Maxime der physischen und psychischen Unversehrtheit der Person begründen lassen (Gabler, 1998, S. 152).

Eine zweite moralische Implikation von Sport verstanden als ein auf Leistungsvergleich angelegtes Unternehmen ist, dass alle an demselben Wettbewerb mit denselben Herausforderungen teilnehmen und damit auch dieselben Chancen auf den Sieg haben (Kretchmar, 2010, S. 104). An einem einfachen Beispiel verdeutlicht: Ein Wettbewerb macht keinen Sinn, wenn manche Teilnehmer*innen zur Bewältigung einer bestimmten Distanz das Rad benutzen dürfen und andere laufen müssen. Man erhielte lediglich Aufschluss darüber, welche Fortbewegungsart die schnellere ist, aber keinen Aufschluss darüber, wer der*die bessere Radfahrer*in bzw. der*die bessere Läufer*in ist. Ein Wettbewerb, der auf den Vergleich spezifischer Fähigkeiten zielt, ist also nur dann möglich, wenn alle Wettbewerbsteilnehmer*innen die gleichen Herausforderungen zu bewältigen haben und mit den gleichen Chancen auf den Sieg an den Start gehen. Wenn nur die sportliche Leistung über Sieg und Niederlage entscheiden soll, dann ist damit das Prinzip derselben Herausforderungen und der Chancengleichheit implizit akzeptiert (Ott, 2004, S. 143). Man sieht an diesem Beispiel, dass Fairness verstanden als Chancengleichheit die Vorbedingung für Leistungsvergleiche im Sport darstellt.

Was bedeutet das Gesagte nun, kurz zusammengefasst, im Hinblick auf Doping? Wenn es beim Sport im Kern um das Austesten spezifischer sportlicher Fähigkeiten gehen soll - und eben nicht um das Austesten der Wirksamkeit von Dopingmitteln - und wenn der Wettbewerb über sportliche Leistung entschieden werden soll - und eben nicht über Biochemie -, dann sollten Dopingmittel verboten werden (Simon, 2001, S. 126).

\section{Potenziale des Sports für die Fair-Play-Erziehung}

Nachdem in den vorangegangenen Abschnitten Grundlagen sportethischen Begründens, Argumentierens und Reflektierens im Zusammenhang 
mit einer internalistischen Konzeption von Fair Play diskutiert wurden, geht es nun um die Frage, ob sich der Sport - wie in der Olympischen Erziehung vorausgesetzt (IOC, 2017) - überhaupt als ein Feld der FairPlay-Erziehung eignet und, wenn ja, worin sein diesbezügliches Potenzial begründet liegt.

Zweifel daran, ob der Sport für Fair-Play-Erziehung geeignet ist, wecken nicht nur zahlreiche Skandale im Hochleistungssport (auch bei Olympischen Spielen), bei denen gefoult, gedopt oder bestochen wurde, sondern auch systematisch erhobene Daten, die nahelegen, dass leistungssportliches Engagement tendenziell nicht zu fairerem Verhalten, sondern - ganz im Gegenteil - zu unfairerem Verhalten führt. So argumentieren etwa Sportler*innen gegenüber Nichtsportler*innen deutlich selbstbezogener und insgesamt betrachtet auf einem niedrigeren Moralniveau (Doty \& Lumpkin, 2010). Prägnant ist des Weiteren, dass viele Athlet"innen den Spitzensport primär mit Leistung, jedoch nur marginal mit Fairness assoziieren (Gaum \& Haut, 2018, S. 15). Dass die Bereitschaft zu unfairem Verhalten mit zunehmendem Alter, steigender Erfahrung im Leistungssport und höherem Leistungsniveau wächst, spricht dafür, dass hier Sozialisationseffekte am Werk sind, die Moral in spezifischer Weise transformieren (Gaum, 2017, S. 357): Eine strikte Ego-, Leistungs- und Erfolgsorientierung stellt sich im engen Kampf um Medaillen für Wettkampfsportler*innen in der Regel als angemessen - weil sportlich erfolgreich heraus (Karvussano et al., 2013). Wichtig ist, dass sich solche Handlungsorientierungen gerade nicht als Konsequenz individueller Moraldefizite einzelner „schwarzer Schafe“ begreifen lassen: Systemtheoretisch betrachtet sind sie vielmehr das erwartbare Resultat von Struktureffekten eines Leistungssportsystems, in dem die Akteur“innen mit zunehmendem Inklusionsgrad ihr Handeln irgendwann so konsequent am Code von Sieg und Niederlage ausrichten, dass von „Überkonformität“ gesprochen werden kann. ${ }^{5}$ Aus pädagogischer Perspektive kommt es zu einer einseitigen Aneignung von Werten in Richtung Egozentrierung, Leistung und Erfolg, die alle sozialerzieherischen Aspekte und Ansprüche überlagert.

5 Zum Begriff der Überkonformität siehe Coakley \& Hughes (1991). Zum Leistungssport aus systemtheoretischer Perspektive siehe Schimank (1988) sowie Stichweh (1990). Zum Doping aus systemtheoretischer Perspektive siehe Bette \& Schimank (2006). 


\begin{abstract}
Moralische Probleme der Sportpraxis als Ausgangspunkte der Fair-PlayErziehung
\end{abstract}

Kann der Sport - vor dem Hintergrund dieser scheinbar gegenteiligen Evidenzen - dennoch ein potenziell fruchtbares Feld der Fair-Play-Erziehung sein? Die genannten Ergebnisse verweisen grundlegend darauf, dass sich Sportler"innen in Wettkampfsituationen stets in einem Spannungsfeld zwischen Leistungsorientierung einerseits und Fairnessverpflichtung andererseits bewegen (Gaum \& Haut, 2018, S. 8). Konkret werden Spiele deswegen „nicht klinisch gespielt“ (Heringer, 1995, S.62), weil alle Spieler"innen - sofern sie ernsthaft spielen - gewinnen möchten und dieses Gewinnstreben potenziell mit Fair-Play-Idealen konfligiert. Gleichzeitig - so lässt sich aufbauend auf den existenzphilosophischen Überlegungen Jean-Paul Sartres und anderer Existentialist*innen folgern - ist moralisches Handeln nur in Situationen möglich, in die sich Menschen nicht selten ohne ihr Zutun "hineingeworfen“ finden (Sartre, 2014, S. 156 ff.). Im Unterschied etwa zu Tieren oder Pflanzen zeichnen sich Menschen in ihrer spezifischen Seinsweise dadurch aus, dass sie zu den jeweiligen Situationen Stellung beziehen können: Sie können sie annehmen oder ablehnen, ihnen verhaftet bleiben oder über sie hinausdenken. Die Geschehnisse der Welt kommen, mit anderen Worten, zwar von außen, zugleich aber können sich Menschen auf ganz unterschiedliche Weise auf sie beziehen, sie unterschiedlich deuten und letztlich entscheiden, etwas zu tun oder nicht zu tun. Relevant im Hinblick auf die Frage der pädagogischen Bedeutung von Sport für die Fair-Play-Erziehung ist hierbei, dass eine Auseinandersetzung mit moralischen Werten stets an konkrete Situationen gebunden ist und sich moralisches Handeln nur in konkreten Situationen realisieren lässt. Darüber hinaus sind diese Situationen im Sport sehr häufig von zwischenmenschlichen Interaktionen geprägt bzw. vollziehen sich in Intersubjektivitätszusammenhängen, die aus Sicht Sartres potenziell konflikthaft sind (Kalthoff, 2015). Genauer betrachtet zeigt sich die Bedeutung moralischer Werte sogar gerade in solchen Situationen, in denen Konflikte und Dilemmata auftauchen und damit Werte zur Disposition stehen.

Begreift man moralische Konfliktlagen und Dilemmasituationen somit als Bedingungen der Möglichkeit von moralischer Reflexion und moralischem Handeln, so stellt sich der Sport als ein hervorragendes Feld für Fair-Play-Erziehung dar: Durch seine agonale Grundstruktur sind Konflikte und Dilemmata im Sport systematisch angelegt - und damit auch zielgerichtet als Reflexionsanlässe mit konkretem Situationsbezug in Moralerziehungsprozesse integrierbar. Die für den Sport typischen 
Wertkonflikte, etwa zwischen Fair Play und Leistung, gilt es dementsprechend als moralisches Bildungspotenzial pädagogisch zu nutzen (Gaum \& Haut, 2018, S. 25). Wenn solche Spannungen somit keine unerwünschten ,Störungen' mehr darstellen, die es zu vermeiden bzw. schnellstmöglich zu eliminieren gilt, sondern vielmehr als willkommene Lerngelegenheiten zu betrachten sind, dann sollten sie sogar gezielt pädagogisch arrangiert und mit den Lernenden ausführlich reflektiert werden, um so die Sinnhaftigkeit von Werten subjektiv erfahrbar und nachvollziehbar zu machen. Das bedeutet, dass bereits in der Planung einer Trainings- oder Unterrichtseinheit bestimmte Situationen von den Lehrenden angelegt werden müssen, die dann - in voller Absicht - in der Durchführung sportethisch relevante Probleme erzeugen. Denn es reicht nicht aus, sich darauf zu verlassen, dass in der Sportpraxis schon von selbst solche Problemsituationen entstehen, die gehaltvolle Diskussionen über Werte ermöglichen. Stattdessen bedarf es hierzu einer expliziten und absichtsvollen Planung (Harvey, Kirk \& O 'Donovan, 2013, S. 107).

\section{Sport als „moralisches Labor"}

Der Spielcharakter des Sports, konkret die weitgehende Konsequenzlosigkeit von Handlungen in einer Welt des "Als ob“ (Bette, 2019, S. 53), begünstigt dabei in besonderer Weise eine Kommunikations- und Diskussionskultur der Offenheit gegenüber abweichenden bzw. unkonventionellen Positionen. Wenn - mit anderen Worten - für die Diskussionsteilnehmer*innen nichts Existenzielles auf dem Spiel steht, erschöpfen sich moralische Kontroversen potenziell weniger leicht im hartnäckigen Festhalten und Durchsetzen von im Vorfeld zementierten Positionen. Vielmehr stehen die Chancen gut für ergebnisoffene und verständigungsorientierte Kommunikation unter der regulativen Idee des ,zwanglosen Zwangs des besseren Arguments“ (Habermas, 1994, S. 370). Des Weiteren gibt die Entlastung von existenziellen Sorgen und Nöten in der Sonderwelt des Sports nicht nur Raum für gedankliches, sondern auch sportpraktisches Experimentieren mit Lösungsansätzen jenseits der eingefahrenen Bahnen. Beispielsweise lassen sich didaktisch begründete Modifikationen von Spielregeln im Sportunterricht verhältnismäßig konsequenzlos erproben - und auch wieder rückgängig machen. In diesem Sinn lässt sich mit den Sportphilosophen Jim Parry (2017) und Graham McFee (2013) dem Sport die Funktion eines „moralischen Labors“ zuschreiben. Parry (2017, S. 144) spricht davon, dass Spiele im Sport „laboratories for value experiments" seien, während McFee (2000, S. 174) einigen Sportarten 
zuschreibt, als „moral laboratories“ fungieren zu können, in denen moralische Konzepte erworben würden, wobei dieser Erwerb jedoch mit weniger Risiko verbunden sei als bei echten moralischen Konfrontationen. Als Raum des Versuchs ohne existenzielle Risiken birgt der Sport die Möglichkeit des unbedenklichen Erprobens moralischer Verhaltensweisen und der absichtsvollen Inszenierung sportethisch relevanter Situationen und Probleme. Nicht zuletzt ist im Sport als einem Feld, das Unbedenklichkeit signalisiert, die Vorläufigkeit, Unsicherheit und Ambiguität von ethischer Erkenntnis potenziell leichter zu akzeptieren als in Feldern, die für Menschen von existenzieller Wichtigkeit sind.

Hingegen führt die relative Konsequenzlosigkeit des Sports nicht dazu, dass es den Sporttreibenden an subjektiver Ernsthaftigkeit, Sinnhaftigkeit und Bedeutsamkeit mangelt, ganz im Gegenteil: In kaum einem anderen Bereich werden Menschen so intensiv mit Kopf (Geist), Herz (Emotionen) und Hand (Körper) angesprochen wie im Sport. Sowohl im Hinblick auf Fair-Play-Erziehung als auch hinsichtlich der lebenslangen Bildung im Bereich von Fairness und Fair-Play ist diese Ganzheitlichkeit kaum zu überschätzen: Wenn sich etwa Spieler*innen in bestimmten Situationen unfair behandelt ,fühlen', so verbleibt dieses Problem nicht im Abstrakten bzw. rein Kognitiven, sondern wurzelt unmittelbar in ganz konkreten, sinnlich-emotionalen Erfahrungen. Wie ein Spiel gespielt wird, nach welchen Regeln und Normen, betrifft die Sporttreibenden somit buchstäblich ,mit Haut und Haaren'. Nicht nur das normative Unterrichtsprinzip der reflektierten Praxis (MSB NRW, 2019, S.9) greift diese Ganzheitlichkeit auf, indem es die sportpraktischen Erfahrungen der Schüler*innen als Anlass zur Reflexion und Diskussion nimmt, um die künftige Sportpraxis nachhaltig zu beeinflussen (Segets, 2012, S. $186 \mathrm{f}$.), das Themenfeld Fairness bietet darüber hinaus auch im außerschulischen lebenslangen Sporttreiben aller Leistungsstufen vielfältige Bildungsmöglichkeiten.

\section{Literatur:}

Bette, K.-H. (2019). Sporthelden: Spitzensport in postheroischen Zeiten. Bielefeld: transcript.

Bette, K.-H. \& Schimank, U. (2006[1995]). Doping im Hochleistungssport. Anpassung durch Abweichung. 2. Aufl., Frankfurt a. M.: Suhrkamp.

Boxill, J. (2003). Introduction: The Moral Significance of Sport. In J. Boxill (Hrsg.), Sports Ethics. An Anthology (S.1-14). Malden, Oxford \& Carlton: Blackwell Publishing. 
Butcher, R. \& Schneider, A. (2001). Fair Play as Respect for the Game. In W. J. Morgan, K. V. Meier \& A. J. Schneider (Hrsg.), Ethics in Sport (S. 21-48). Champaign: Human Kinetics.

Campos, D. G. (2014). Skills. In C. R. Torres (Hrsg.), The Bloomsbury Companion to the Philosophy of Sport (S. 368-370). London, Oxford, New York, New Delhi \& Sydney: Bloomsbury.

Carr, C. L. (2000). On Fairness. Aldershot: Engl.-Burlington.

Caysa, V. (2004). Was ist ein fairer Umgang mit dem Körper? In C. Pawlenka (Hrsg.), Sportethik. Regeln - Fairness - Doping (S. 149-163). Paderborn: Mentis.

Coakley, J. \& Hughes, R. (1991). Positive Deviance Among Athletes. The Implications of Overconformity Among Athletes. Sociology of Sport Journal, 8, 307-325.

Court, J. \& Gerhardt, V. (1992). Sportethik. In P. Röthig (Hrsg.), Sportwissenschaftliches Lexikon (S. 428-429). Schorndorf: Hofmann.

D'Agostino, F. (1981). The Ethos of Games. Journal of the Philosophy of Sport, 8 (1), 7-18.

Dietrich, J. (2009). Die Kraft der Konkretion oder: Die Rolle deskriptiver Annahmen für die Anwendung und Kontextsensitivität ethischer Theorie. Ethik in der Medizin, 21 (3), 213-221.

Doty, J. \& Lumpkin, A. (2010). Do sports build or reveal character? An exploratory study at one service academy. The Physical Educator, 67 (1), 18-32.

Fraleigh, W. P. (2014). Cheating. In C. R. Torres (Hrsg.), The Bloomsbury Companion to the Philosophy of Sport (S.341-343). London, Oxford, New York, New Delhi \& Sydney: Bloomsbury.

Gabler, H. (1998). Fairneß / Fair Play. In O. Grupe \& D. Mieth (Hrsg.), Lexikon der Ethik im Sport (S. 149-158). Schorndorf: Hofmann.

Gaffrey, P. (2015). Competition. In M. McNamee \& W. J. Morgan (Hrsg.), Routledge Handbook of the Philosophy of Sport (S. 287-299). London \& New York: Routledge.

Gaum, C. (2017). Fairnessverständnis im Amateurfußball. German Journal of Exercise and Sport Research, 47 (4), 348-359.

Gaum, C. \& Haut, J. (2018). Sportliche Vorbilder? Welche Werte durch Spitzensportler (nicht) vermittelt werden. Zeitschrift für sportpädagogische Forschung, 6 (1), 5-28.

Gerhardt, V. (1997). Die Moral des Sports. In V. Caysa (Hrsg.), Sportphilosophie (S. 172-202). Leipzig: Reclam.

Gleaves, J. (2014). Rules. In C. R. Torres (Hrsg.), The Bloomsbury Companion to the Philosophy of Sport (S. 368-370). London, Oxford, New York, New Delhi \& Sydney: Bloomsbury.

Grupe, O. (2013). Die Olympische Idee ist eine „Erziehungsidee“. In M. Krüger \& A. Hofmann (Hrsg.), Olympia als Bildungsidee. Beiträge zur olympischen Geschichte und Pädagogik (S. 9-22). Wiesbaden: Springer VS.

Guttmann, A. (1987). Ursprünge, soziale Basis und Zukunft des Fair Play. Sportwissenschaft, 17 (1), 7-16. 
Günter, S. (2005). Geschlechterkonstruktion im Sport. Eine historische Untersuchung der nationalen und regionalen Turn- und Sportbewegung des 19. und 20. Jahrhunderts. Göttingen: Cuvillier.

Habermas, J. (1994). Faktizität und Geltung. Beiträge zur Diskurstheorie des Rechts und des demokratischen Rechtsstaats. Frankfurt a. M.: Suhrkamp.

Harvey, S., Kirk, D. \& O’Donovan, T. M. (2013). Sport Education as pedagogy for promoting sport as ethical practice. In S. Harvey \& R. L. Light (Hrsg.), Ethics in youth sport. Policy and pedagogical applications (S.107-121). London \& New York: Routledge.

Heringer, H. J. (1995). Fairness und Moral. In V. Gerhardt \& M. Lämmer (Hrsg.), Fairness und Fairplay (S. 55-68). Sankt Augustin: Academia.

International Olympic Committee [IOC] (Hrsg.). (2017). Delivering OVEP Playbook. A Practical Guide to Olympic Values Education. Lausanne: IOC. Abgerufen am 2.1.2021 unter https://library.olympic.org/Default/doc/SYRA CUSE/207025/delivering-ovep-playbook-a-practical-guide-to-olympic-values-educ ation-train-the-trainers-internatio.

Kalthoff, J. (2015). Knechtschaft im Sportunterricht. Ein existentialistischer Blick auf die Leistungsbeurteilung. In E. Hübner \& K. Reinhart (Hrsg.), Sport Geschichte - Pädagogik. Festschrift zum 60. Geburtstag von Michael Krüger (S. 320331). Hildesheim: Arete.

Kavussanu, M., Boardly, I. D., Sagar, S. S. \& Rind, C. (2013). Bracketed morality revisited: how do athletes behave in two contexts? Journal of Sport \& Exercise Psychology, 35, 449-463.

Kähler, R. (1985). Moralerziebung im Sportunterricht: Untersuchung zur Regelpraxis und zum Regelbewusstsein. Thun: Deutsch.

Kretchmar, R. S. (2010). From test to contest: An analysis of two kinds of counterpoint in sport. In M. McNamee (Hrsg.), The Ethics of Sports. A Reader (S. 100105). New York: Routledge.

Lenk, H. (1972). Werte, Ziele, Wirklichkeit der modernen Olympischen Spiele. Schorndorf: Hofmann.

Lenk, H. (2004). Wettkampf-Fairness, assoziative Moral und strukturelle DilemmaSituationen. In C. Pawlenka (Hrsg.), Sportethik. Regeln - Fairness - Doping (S. 119-132). Paderborn: Mentis.

Llewellyn, M. P. \& Gleaves, J. (2016). The Rise and Fall of Olympic Amateurism. Urbana, Chicago \& Springfield: University of Illinois Press.

Loland, S. (2010). Fairness in Sport: An Ideal and its Consequences. In M. McNamee (Hrsg.), The Ethics of Sports. A Reader (S. 116-124). London \& New York: Routledge.

López Frías, F. J. (2017). Broad Internalism and Interpretation: A Plurality of Interpretivist Approaches. In S. E. Klein (Hrsg.), Defining Sport. Conceptions and Borderlines (S. 22-44). Lanham, Boulder, New York \& London: Lexington Books. 
McFee, G. (2000). Spoiling: An Indirect Reflection of Sport's Moral Imperative? In T. Tannsjo and C. Tamburrini (Hrsg.), Values in Sport (S. 172-182). London: Routledge.

McFee, G. (2013). Olympism and Sport's Intrinsic Value. In M. McNamee \& J. Parry (Hrsg.), Olympic Ethics and Philosophy (S. 109-129). London \& New York: Routledge.

Meinberg, E. (1998). Sportethik / Moral des Sports. In O. Grupe \& D. Mieth (Hrsg.), Lexikon der Ethik im Sport (S. 498-504). Schorndorf: Hofmann.

Ministerium für Schule und Bildung des Landes Nordrhein-Westfalen [MSB NRW] (Hrsg.). (2019). Kernlehrplan für die Sekundarstufe I Gymnasium in Nordrhein-Westfalen. Sport. Düsseldorf: o.V. Abgerufen am 27.1.2021 unter https://www.schulentwicklung.nrw.de/lehrplaene/lehrplan/210/g9_sp_klp_ 3426_2019_06_23.pdf.

Morgan, W. J. (2012). Broad Internalism, Deep Conventions, Moral Entrepreneurs, and Sport. Journal of the Philosophy of Sport, 39 (1), 65-100.

Morgan, W. J. (2015). Conventionalism and Sport. In M. McNamee \& W. J. Morgan (Hrsg.), Routledge Handbook of the Philosophy of Sport (S.35-52). Routledge: London \& New York.

Müller, N. (1998). Olympische Erziehung. In O. Grupe \& D. Mieth (Hrsg.), Lexikon der Ethik im Sport (S. 385-395). Schorndorf: Hofmann.

Ott, K. (2004). Grundelemente der Gerechtigkeit im Sport. In C. Pawlenka (Hrsg.), Sportethik. Regeln - Fairness - Doping (S. 133-147). Paderborn: Mentis.

Parry, J. (2017[1986]). Values in Physical Education. In P. Tomlinson \& M. Quinton (Hrsg.), Values Across the Curriculum (S. 134-157). 2. Aufl., London: Routledge.

Pieper, A. (2017[1985]). Einführung in die Ethik. 7. Aufl. Tübingen: Francke.

Pilz, G. (1995). Zum Problem struktureller Bedingungen für Unfairneß. Eine empirische Analyse. In V. Gerhardt \& M. Lämmer (Hrsg.), Fairness und Fair Play (S. 173-201). Sankt Augustin: Academia.

Reinold, M. (2016). Doping als Konstruktion. Eine Kulturgeschichte der Anti-DopingPolitik. Bielefeld: transcript.

Reinold, M. \& Kalthoff, J. (2019). Werte und Regeln: Fairplay. In A. Güllich \& M. Krüger (Hrsg.), Sport in Kultur und Gesellschaft. Handbuch Sport und Sportwissenschaft (S. 1-16). Berlin \& Heidelberg: Springer. https://doi.org/10.1007/978-3-662 -53385-7_27-2.

Russell, J. S. (1999). Are Rules All An Umpire Has to Work with? Journal of the Philosophy of Sport, 26 (1), 27-49.

Russell, J. S. (2014). Competitive Sport, Moral Development and Peace. In C. R. Torres (Hrsg.), The Bloomsbury Companion to the Philosophy of Sport (S. 228-244). London, Oxford, New York, New Delhi \& Sydney: Bloomsbury.

Sandel, M. J. (2020). Vom Ende des Gemeinwohls. Wie die Leistungsgesellschaft unsere Demokratien zerreißt. 2. Aufl. Frankfurt a. M.: Fischer. 
Sartre, J.-P. (2014). Der Existentialimus ist ein Humanismus. In J.-P. Sartre, Der Existenzialismus ist ein Humanismus und andere philosophische Essays (S. 145-192). Reinbek: Rowohlt.

Schimank, U. (1988). Die Entwicklung des Sports zum gesellschaftlichen Teilsystem. In R. Mayntz, B. Rosewitz, U. Schimank \& R. Stichweh (Hrsg.), Differenzierung und Verselbstständigung. Zur Entwicklung gesellschaftlicher Teilsysteme (S. 181-232). Frankfurt \& New York: Campus.

Segets, M. (2012). Sportphilosophie in der Schule. Zur didaktischen Transformation sportphilosophischer Themen für den Unterricht der Fächer „Philosophie“/"Praktische Philosophie“ und „Sport“. Berlin: Lit.

Siep, L. (1995). Arten und Kriterien der Fairness im Sport. In V. Gerhardt \& M. Lämmer (Hrsg.), Fairness und Fairplay (S. 87-103). Sankt Augustin: Academia.

Simon, R. L. (2000). Internalism and Internal Values in Sport. Journal of the Philosophy of Sport, 27 (1), 1-16.

Simon, R. L. (2001). Good Competition and Drugs-Enhanced Performance. In W. J. Morgan, K. V. Meier \& A. J. Schneider (Hrsg.), Ethics in Sport (S. 119-129). Champaign: Human Kinetics.

Simon, R. L. (2010). Fair Play. The Ethics of Sport. Boulder: Westview Press.

Simon, R. L. (2014). Theories of Sport. In C. R. Torres (Hrsg.), The Bloomsbury Companion to the Philosophy of Sport (S. 83-97). London, Oxford, New York, New Delhi \& Sydney: Bloomsbury.

Simon, R. L. (2015). Internalism and Sport. In M. McNamee \& W. J. Morgan (Hrsg.), Routledge Handbook of the Philosophy of Sport (S. 22-33). London \& New York: Routledge.

Stichweh, R. (1990). Sport - Ausdifferenzierung, Funktion, Code. Sportwissenschaft, 20, 373-389.

Suits, B. (2004). Was ist ein Regelspiel? In C. Pawlenka (Hrsg.), Sportethik. Regeln Fairness - Doping (S. 29-41). Paderborn: Mentis.

Tetens, H. (2006). Philosophisches Argumentieren. Eine Einführung. München: Beck.

Torres, C. R. (2000). What Counts as Part of a Game? A look at skills. Journal of the Philosophy of Sport, 27 (1), 81-92.

Triviño, J. (2014). Formalism. In C. R. Torres (Hrsg.), The Bloomsbury Companion to the Philosophy of Sport (S. 358-359). London, Oxford, New York, New Delhi \& Sydney: Bloomsbury. 
